\title{
8p23.1 duplication syndrome; a novel genomic condition with unexpected complexity revealed by array $\mathrm{CGH}$
}

John CK Barber*,1,2,3, Viv K Maloney ${ }^{1}$, Shuwen Huang ${ }^{1}$, David J Bunyan ${ }^{2}$, Lara Cresswell ${ }^{4}$, Esther Kinning ${ }^{4}$, Anna Benson ${ }^{5}$, Tim Cheetham ${ }^{5}$, Jonathan Wyllie ${ }^{6}$, Sally Ann Lynch ${ }^{5}$, Simon Zwolinski ${ }^{5}$, Laura Prescott ${ }^{7}$, Yanick Crow ${ }^{8}$, Rob Morgan ${ }^{7}$ and Emma Hobson ${ }^{8}$

\footnotetext{
${ }^{1}$ National Genetics Reference Laboratory (Wessex), Salisbury NHS Foundation Trust, Salisbury, Wiltshire, UK; ${ }^{2}$ Wessex Regional Genetics Laboratory, Salisbury NHS Foundation Trust, Salisbury, Wiltshire, UK; ${ }^{3}$ Human Genetics Division, Southampton University School of Medicine, Southampton General Hospital, Southampton, UK; ${ }^{4}$ Leicestershire Genetics Centre, Leicester Royal Infirmary, Leicester; ${ }^{5}$ Institute of Human Genetics, International Centre for Life, Central Parkway, Newcastle upon Tyne, UK; ${ }^{6} J a m e s$ Cook University Hospital, Marton Road, Middlesbrough, Cleveland, UK; ${ }^{7}$ Regional Cytogenetics Unit, St James Hospital, Beckett Street, Leeds, UK; ${ }^{8}$ Clinical Genetics, St James Hospital, Beckett Street, Leeds, UK
}

The 8p23.1 deletion syndrome is established but not an equivalent duplication syndrome. Here, we report five patients; a de novo prenatal case and two families in which 8p23.1 duplications have been directly transmitted from mothers to children. Dual-colour fluorescent in situ hybridisation, multiplex ligationdependent probe amplification analysis and customised oligonucleotide array comparative genomic hybridisation (oaCGH) indicated an $\sim 3.75 \mathrm{Mb}$ duplication of most of band 8p23.1 between the olfactory receptor/defensin repeats (ORDRs) in all cases. However, oaCGH revealed an additional duplication of $500 \mathrm{~kb}$ adjacent to the proximal ORDR in Family 1 and an additional deletion of $3.14 \mathrm{Mb}$ within the Nablus Mask-Like Facial Syndrome region of 8q22.1 in Family 2. Copy number variation at introns 4-5 of the GATA4 gene was also identified. This $8 \mathrm{p} 23.1$ duplication syndrome is associated with a characteristic facial phenotype including a prominent forehead and arched eyebrows. Adrenal insufficiency, Tetralogy of Fallot, partial 2/3 syndactyly of the toes and cleft palate in some individuals may be explained by ascertainment bias, incomplete penetrance and/or the presence of the microdeletion in Family 2 . The duplication is compatible with normal early childhood development but, although our adult cases live independent lives with varying degrees of support, learning difficulties have been experienced by some family members. We conclude that the 8p23.1 duplication syndrome is a genomic condition with an emerging but variable phenotype that may be under-diagnosed. Our results demonstrate that direct transmission does not distinguish genuine duplications from euchromatic variants and illustrate the power of array CGH to reveal unexpected additional imbalances in affected patients.

European Journal of Human Genetics (2008) 16, 18-27; doi:10.1038/sj.ejhg.5201932; published online 17 October 2007

Keywords: duplication 8p23.1; microdeletion 8q22.1; genomic condition

*Correspondence: Dr JCK Barber, Wessex Regional Genetics Laboratory, Salisbury District Hospital, Salisbury, Wiltshire SP2 8B], UK.

Tel: + 44 (0)1722 429080; Fax: + 44 (0)1722 338095;

E-mail: john.barber@salisbury.nhs.uk

Received 3 July 2007; revised 23 August 2007; accepted 30 August 2007; published online 17 October 2007
Introduction

Deletion of 8 p23.1 between the 8 p23.1 olfactory receptor/ defensin repeats (ORDRs) (REPeat Proximal (REPP) and REPeat Distal (REPD) ${ }^{1}$ ) is an established genomic disorder 
associated with developmental delay, behavioural problems, congenital heart disease, diaphragmatic hernia and a Fryns syndrome-like condition. ${ }^{2-4}$ Despite the use of tiling path and targeted microarray CGH in large numbers of patients, ${ }^{5,6}$ the equivalent duplication has, to our knowledge, only been mapped with fluorescent in situ hybridisation (FISH) and reported once in a patient ascertained with mild language delay and pulmonary stenosis. $^{7}$ This duplication between REPP and REPD alternated between three copies of a 300-450 kb segment flanking but distal to REPD. Distal $8 p$ is also remarkable for a variety of other complex chromosome anomalies, ${ }^{1,7-10}$ for a relatively high number of families in which imbalances are transmitted directly from parents to children $^{11}$ and for the degree of copy number polymorphism associated with the defensin gene clusters. ${ }^{7,12-16}$ Here we present a prenatal case and two families with transmitted interstitial duplications of $8 \mathrm{p} 23.1$ that are associated with a common but variable phenotype and unexpected complexity identified using oligonucleotide array comparative genomic hybridisation (oaCGH).

\section{Methods \\ Cytogenetics, molecular cytogenetics and molecular genetics}

G-banded chromosomes were analysed at the 550 or higher band level and FISH carried out with Ensembl $1 \mathrm{Mb}$ and/or $37 \mathrm{k}$ cloneset bacterial artificial chromosomes (BACs) (Table 1). Genes assigned to the deleted regions were obtained from the Ensembl database using the MartView data export tool.

Multiplex ligation-dependent probe amplification analysis (MLPA) was carried out with probe sets PO36 and P139 using the standard protocol of MRC-Holland. ${ }^{17}$ Probe set PO36 was combined with three additional in-house MLPA probes corresponding to the MFHAS1 (MASL1), DEFB1 and GATA4 genes (PO36 + ; sequences available upon request) (Table 1).

Oligonucleotide array comparative genomic hybridisation was carried out using test genomic DNAs obtained using a standard salt extraction method. Reference DNA was normal human male genomic DNA purchased from Promega Corporation (G147A 19813601, Madison, WI, USA). DNA quantity and quality were assessed by UV/Vis spectrophotometry and agarose gel analysis. A selfdesigned $4 \times 44 \mathrm{~K}$ custom array (NGRL WESSEX CONSTITUTIONAL ARRAY CGH V1, design \# 015543, Agilent) was applied to detect genome-wide copy number changes. In brief, patient and reference DNAs were digested with AluI and $R s a \mathrm{I}$ and the restriction enzymes inactivated by incubation at $65^{\circ} \mathrm{C}$. The Cy5- and Cy3-labelled DNA sample pair were combined and mixed with human Cot1 DNA. Prior to hybridisation, the samples were heated at $95^{\circ} \mathrm{C}$ for $3 \mathrm{~min}$ and then incubated for $30 \mathrm{~min}$ at $37^{\circ} \mathrm{C}$.
Labelled target solution was hybridised to the $4 \times 44 \mathrm{~K}$ array with three other samples using SureHub chambers (G2534A) in a $65^{\circ} \mathrm{C}$ rotisserie oven (G2545A) set to rotate at $20 \mathrm{r} . p . m$. for $24 \mathrm{~h}$. After hybridisation, the slide was washed and dried according to the Agilent Oligonucleotide Array-based CGH for Genomic DNA Analysis protocol Version 4.0 (G4140-90010). The microarray slide was scanned immediately using an Agilent microarray scanner (G2565BA). Image and data analyses were performed using the Agilent CGHAnalytics (v3.4) microarray software.

\section{Clinical histories \\ Case 1}

A female patient was referred for amniocentesis at the age of 33 after biochemical screening had indicated a 1 in 150 risk of Down syndrome. A female child with a duplication of the short arm of chromosome 8 was found and the parents elected to continue with the pregnancy. There were no complications during pregnancy and, after a normal delivery at 40 weeks gestation, apgars were 8 at $1 \mathrm{~min}$ and 9 at $5 \mathrm{~min}$. Birth weight was $3.15 \mathrm{~kg}$ and occipitofrontal circumference (OFC) $33.6 \mathrm{~cm}$ (10th centile). At 15 months of age, this girl had a length of $75 \mathrm{~cm}(10-50$ th centile), OFC of $46.6 \mathrm{~cm}$ (50th centile) and a weight of $9.34 \mathrm{~kg}$ (1050 th centile). She had only subtle facial features such as a prominent forehead, mildly arched eyebrows and slightly upward slanting palpebral fissures (Figure 1a and b). She appears to be developing well, is walking around the furniture and able to say a few words. Neurological examination at 15 months was normal as was an echocardiogram of the heart.

Family 1 A female infant was born at 42 weeks gestation by emergency caesarean section for failure to progress. Her mother had an ovarian cyst excised in pregnancy but antenatal scans were otherwise unremarkable. Her birth weight was $3.6 \mathrm{~kg}$ and she resembled her mother at delivery. In the first hours of life, the proband was unwell with hypothermia, hypotension, poor oxygen saturation, hypoglycaemia, hyponatraemia and seizures. Adrenal imaging was abnormal, she had profound salt-wasting and required mineralocorticoid and glucocorticoid replacement. Subsequent investigations confirmed primary adrenal insufficiency with large adrenal glands and she was commenced on adrenal hormone replacement. An echocardiogram demonstrated Tetralogy of Fallot (TOF), which was thought to account in part for her profound hypotensiveness and cyanosis. There was qualitative and quantitative evidence of sulphite oxidase deficiency but normal sulphite oxidase activity was found in fibroblasts.

On examination at 4 months of age, she was microcephalic (HC $38 \mathrm{~cm},<0.4$ th centile) with Cushingoid facies, a carp-shaped mouth, a high-arched palate, round ears, proximally placed thumbs bilaterally, long fingers, 
Table 1 BAC FISH, MLPA and oaCGH results in Case 1 (C1), Family 1 (F1) and Family 2 (F2)

\begin{tabular}{|c|c|c|c|c|c|c|c|}
\hline 1. Band & $\begin{array}{l}\text { 2. BAC/MLPA*/ } \\
\text { aCGH (Build 35) }\end{array}$ & 3. Start and stop (Mb from telomere) & 4. C1 proband & 5. F1 proband & 6. F1 mother & 7. F2 proband & 8. F2 mother \\
\hline 8p23.2 & CSMD1 (4 probes)* & $2782789-4839736$ & ND & Normal & Normal & Normal & Normal \\
\hline $8 p 23.2$ & $336 N 16$ & $2888584-3078882$ & ND & Normal & ND & ND & ND \\
\hline $8 p 23.2$ & $16 \mathrm{H} 11$ & $4177855-4396741$ & ND & Normal & ND & ND & ND \\
\hline $8 p 23.2$ & RP5-991O23 & $5316020-5469277$ & ND & Normal & Normal & Normal & Normal \\
\hline $8 \mathrm{p} 23.1$ & CTD-2629I16 & $6674740-6695317$ & ND & Normal & Normal & Normal & Normal \\
\hline $8 p 23.1$ & ANGPT2* & $6347601-6408172$ & ND & Normal & Normal & Normal & Normal \\
\hline $8 p 23.1$ & DEFB1 (2 probes)* & $6715511-6722939$ & ND & Normal & Normal & Normal & Normal \\
\hline $8 p 23.1$ & DEFA6 (2 probes)* & $6769631-6771008$ & ND & Normal & Normal & Normal & Normal \\
\hline $8 p 23.1$ & DEFA4 (2 probes)* & $6780755-6783196$ & ND & Normal & Normal & Normal & Normal \\
\hline 8 p23.1 & $D E F A 5^{*}$ & $6900239-6901669$ & ND & Normal & Normal & Normal & Normal \\
\hline $8 \mathrm{p} 23.1$ & $\mathrm{aCGH}$ & 6907624 & ND & ND & Normal & Normal & Normal \\
\hline REPD & $\mathrm{aCGH}$ & 7256229 & ND & ND & Dup & Del & Del \\
\hline REPD & $112 \mathrm{~N} 11$ & $7367548-7578862$ & Dup & Enh & Enh & Normal & Normal \\
\hline REPD & $\begin{array}{l}\text { DEFB4 etc (10 } \\
\text { probes)* }\end{array}$ & Complex & ND & Dup & Dup & Del & Normal \\
\hline REPD & $\mathrm{aCGH}$ & 7789937 & ND & ND & Dup & Del & Del \\
\hline REPD & $\mathrm{aCGH}$ & 8168040 & ND & ND & Dup & Dup & Dup \\
\hline $8 p 23.1$ & $211 C 9$ & $8479797-8687720$ & Dup & Dup & Dup & Dup & Dup \\
\hline $8 p 23.1$ & MFHAS1 (MASL1)* & $8680942-8787978$ & ND & Dup & Dup & Dup & Dup \\
\hline $8 \mathrm{p} 23.1$ & $P P P 1 R 3 B^{*}$ & $9032916-9045616$ & ND & Dup & Dup & Dup & Dup \\
\hline $8 \mathrm{p} 23.1$ & TNKS* & $9450855-9671801$ & ND & Dup & Dup & Dup & Dup \\
\hline $8 p 23.1$ & $M S R A^{*}$ & $9949240-10323808$ & ND & Dup & Dup & Dup & Dup \\
\hline $8 \mathrm{p} 23.1$ & $112 \mathrm{G} 9$ & $10028624-10236504$ & ND & Dup & Dup & Dup & Dup \\
\hline $8 \mathrm{p} 23.1$ & GATA4* & $11599162-11654918$ & ND & Dup & Dup & Dup & Dup \\
\hline $8 p 23.1$ & GATA4 aCGH & 11604907 & ND & ND & Trp & Trp & Trp \\
\hline $8 \mathrm{p} 23.1$ & 589N15 & $11626380-11804128$ & Dup & Dup & Dup & Dup & Dup \\
\hline $8 \mathrm{p} 23.1$ & $\mathrm{aCGH}$ & 11897580 & ND' & ND' & Dup & Dup & Dup \\
\hline REPP & $\mathrm{aCGH}$ & 12284988 & ND & ND & Dup & Del & Del \\
\hline REPP & 24D9 & $12433488-12590982$ & Dup & Enh & Enh & ND & ND \\
\hline $8 p 23.1$ & $\mathrm{aCGH}$ & 12626674 & ND & ND & Trp & Normal & Normal \\
\hline $8 p 22$ & $92 \mathrm{C} 1$ & $12880230-13024002$ & ND & Dup & Dup & Normal & Normal \\
\hline $8 p 22$ & $\mathrm{aCGH}$ & 13127400 & ND & ND & Dup & Normal & Normal \\
\hline $8 p 22$ & $\mathrm{aCGH}$ & 13207141 & ND & ND & Normal & Normal & Normal \\
\hline $8 p 22$ & $433 \mathrm{~L} 7$ & $14277096-14462154$ & Normal & Normal & Normal & Normal & Normal \\
\hline $8 p 22$ & MSR1* & $16009761-16094595$ & Normal & Normal & Normal & ND & ND \\
\hline $8 p 21.3$ & CGAT1* & $19305952-19584552$ & Normal & Normal & Normal & ND & ND \\
\hline $8 q 22.1$ & $\mathrm{aCGH}$ & 93214935 & ND & ND & Normal & Normal & Normal \\
\hline $8 q 22.1$ & $\mathrm{aCGH}$ & 93458057 & ND & ND & Normal & Del & Del \\
\hline $8 q 22.1$ & 100L22 & $93711455-93899438$ & ND & ND & ND & Del & Del \\
\hline $8 q 22.1$ & $10 N 23$ & $94720059-94916025$ & ND & ND & ND & Del & Del \\
\hline $8 q 22.1$ & $\mathrm{aCGH}$ & 96599593 & ND & ND & Normal & Del & Del \\
\hline $8 q 22.1$ & $\mathrm{aCGH}$ & 96734993 & ND & ND & Normal & Normal & Normal \\
\hline
\end{tabular}

Notes: column 1: G-dark bands in bold; column 2: all BACs RP11 unless indicated, "MRC Holland defensin kit P139; column 3: all base pairs derived from Build 35 of the human genome; columns 4-8: C1, Case 1, F1, family 1, F2, family 2, bold type and grey background indicates extent of imbalances.

Abbreviations: BAC, bacterial artificial chromosomes; del, deleted; dup, duplicated; enh, enhanced; FISH, fluorescent in situ hybridisation; MLPA, multiplex ligation-dependent probe amplification analysis; ND, no data; oaCGH, oligonucleotide array comparative genomic hybridisation; trp, triplicated. 
Family 1
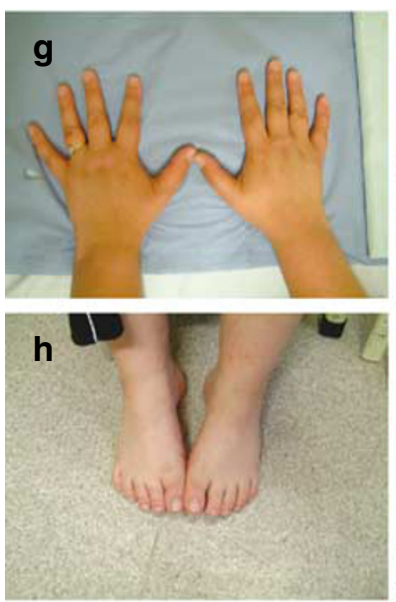

Case 1
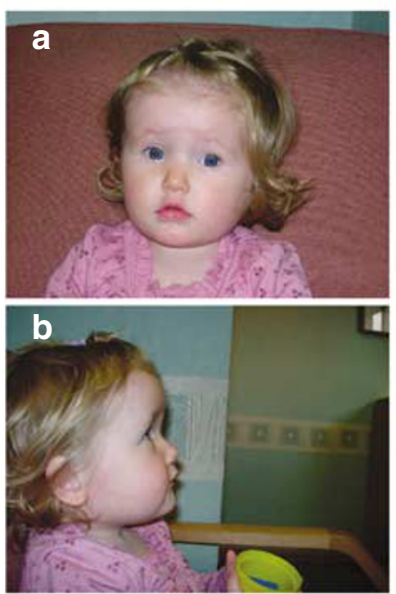
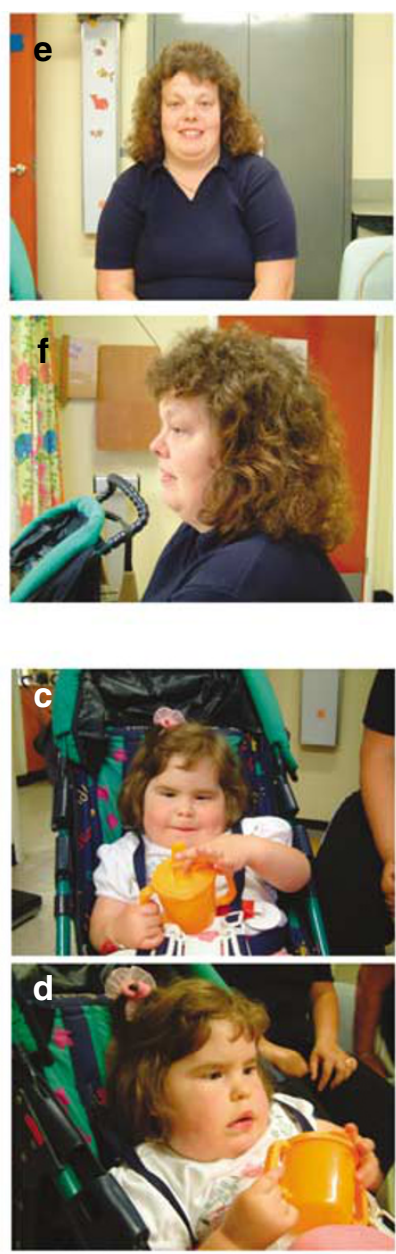

\section{Family 2}
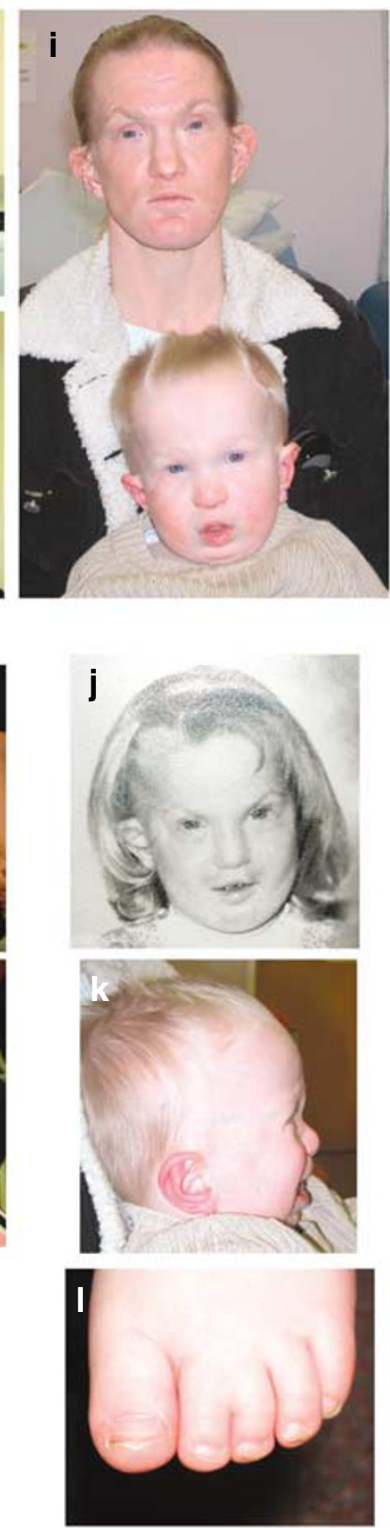

Figure 1 (a-l) Phenotype of Case 1 and Families 1 and 2: (a) portrait and (b) profile of Case 1; (c) portrait and (d) half-profile of the proband from Family $1 ;(\mathbf{e})$ portrait and (f) profile of the mother from Family $1 ;(\mathbf{g}-\mathbf{h})$ hands and toes of the mother from Family $1 ;$ (i) portrait of proband and mother from Family 2; (j) portrait of mother from Family 2 as a child; (k) profile of the proband from Family 2; (I) partial 2/3 syndactyly of the toes in the proband from Family 2. Please note the characteristic facial appearance including the prominent forehead and arched eyebrows in the majority of patients.

broad thumbs and big toes (Figure 1c and d). By 3 years 9 months, her head circumference was $49 \mathrm{~cm}$ (between the 10th and 25th centiles), her height $92.3 \mathrm{~cm}$ ( $\sim 2$ nd centile) and her weight $15.5 \mathrm{~kg}$ (between the $25 \mathrm{th}$ and $50 \mathrm{th}$ centiles). At 4 years of age, the TOF has been repaired. She has cerebral palsy that is thought to be related to the hypoxic-ischaemic insult in early life but is generally well on adrenal steroid hormone replacement. At birth she had been described as having a prominent forehead and arched eyebrows (data not shown), but these features were not evident by the age of four although present in her mother.

The proband's mother has mild dysmorphic features including frontal bossing and coarse facial features (Figure $1 \mathrm{e}$ and $\mathrm{f}$ ). She has learning difficulties but gained five ' $\mathrm{O}$ ' levels at school. She had no syndactyly of either hands or feet (Figure $1 \mathrm{~g}$ and $\mathrm{h}$ ). There were no grounds to suspect a 
heart defect and an echocardiogram has not been carried out. The proband's father also has learning difficulties but both mother and father are in employment.

Family 2 The proband was born at $40+5$ weeks gestation with a birth weight of $3.39 \mathrm{~kg}$ (25th centile and head circumference of $35 \mathrm{~cm}$ (25-50th centile). He was referred at 11 weeks of age because of dysmorphism and a family history of dysmorphism and sudden infant death. He had similar facial features to his mother with a prominent forehead, deep-set eyes and blepharophimosis (Figure 1i, j and $\mathrm{k}$ ). His head circumference was $39.5 \mathrm{~cm}$ (25th centile) and his eyebrows were neat and arched (Figure 1i). His mouth was small, his nasal tip bulbous and his ears low-set and dsyplastic (Figure $1 \mathrm{i}$ and $\mathrm{k}$ ). Both mother and son had partial 2/3 toe syndactyly (Figure 11). He had walked at 18 months of age and his development was minimally delayed when he was reviewed at 22 months of age. At $49 \mathrm{~cm}$, his head circumference remained on the 25th centile, his weight was $12.1 \mathrm{~kg}$ (50th centile) and his height was $79.5 \mathrm{~cm}$ (between the 2nd and 25th centiles). He had a fine intention tremor.

The proband's mother lives independently although she does have support from her family. In addition to her facial features, she was born with a cleft palate and has had surgery to bring her jaw forward. The proband had two maternal half-siblings. The first was a boy who died at 7 weeks of sudden infant death at home. He had not been referred to Clinical Genetics but a post-mortem report detailed similar facial features to the proband and his mother and, in addition, a cleft of the soft palate, persistent ductus arteriosus, bowel malrotation and inguinal hernia. The death was attributed to cyclomegalovirus infection. The second half-sibling was a healthy girl.

\section{Results}

Cytogenetics and molecular cytogenetics

Case 1 G-banded analysis of amniotic fluid cultures showed a duplication of the distal short arm of chromosome 8 in the fetus (Figure 2a). Dual-colour FISH with BACs RP11-211C9 and RP11-589N15, which map to either end of the interval between the ORDRs, gave consistently larger signals on one chromosome 8 in metaphases (Figure 3a) and clear evidence of duplicated signals in interphase nuclei (Figure 3a). BACs RP11-122N11 and RP11-24D9, which map to the distal (REPP) and proximal (REPD) ORDRs respectively, also gave larger signals which suggest that the variable defensin regions may have been included in the duplication (data not shown). The karyotype of the proband was 46,XX, dup(8)(p23.1p23.1).ish dup(8)(p23.1 p23.1) $(122 \mathrm{~N} 11++?+, 211 \mathrm{C} 9++, 589 \mathrm{~N} 15++, 24 \mathrm{D} 9++?+)$. DNA was not available from this infant for MLPA and/or oaCGH testing.

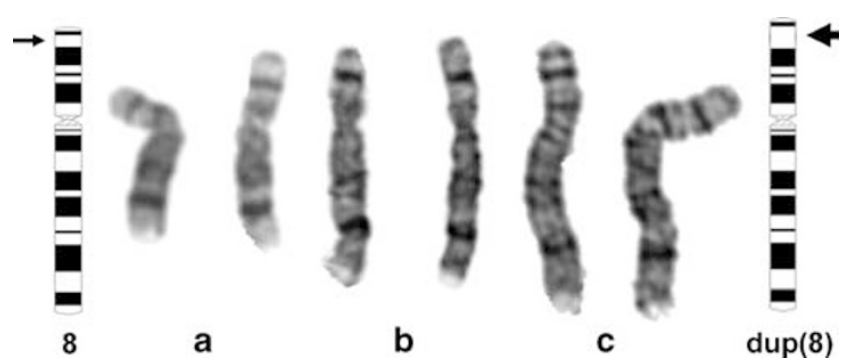

Figure 2 (a-c) Partial karyotypes of (a) Case 1, (b) the mother from Family 1 and (c) the mother from Family 2. The duplicated chromosome is on the right-hand side of each pair of chromosomes. The slim arrow indicates band 8p23.1 on the normal idiogram and the broad black arrow indicates the expanded size of this band on the duplicated idiogram.

Family 1 Five index cases with extra material in 8p23.1 were retrospectively tested with FISH in Newcastle to distinguish euchromatic variants from genuine duplications. G-banded analysis of peripheral blood cultures showed a duplication of the distal short arm of chromosome 8 in one of the five index cases as well as in her mother (Figure 2b). A microdeletion of 22q11.2 was excluded in the proband using conventional FISH with a probe for TUPLE1. Dual-colour FISH showed that BACs between REPP and REPD were duplicated (Table 1; Figure $3 \mathrm{~b}$ and $\mathrm{c}$ ) while the REPD BAC 122N11 gave enhanced signal strength (enh) at the variable defensin locus (data not shown) and the REPP BAC 24D9 was present in three copies which alternated with the duplication (Figure $3 \mathrm{~b}$ and c). All other probes gave normal results.

Oligonucleotide array comparative genomic hybridisation in the mother not only confirmed duplication of the interval between REPP and REPD but also showed a further triplication extending from REPP in 8p23.1 to introns 5-6 of the DLC1 gene in 8p22 (Figure 4a; Table 1). Only duplication was confirmed in both mother and daughter using FISH with BAC RP11-92C1 (Figure 3d; Table 1). In addition, the flanking ORDRs were increased in copy number relative to the control and a copy number polymorphism within introns $4-5$ of the GATA4 gene was identified with the oligonucleotide mapping to base pair 11604907 . No other significant gains or losses were found. Grandparental chromosomes were normal and this duplication was therefore de novo in the mother. However, there was no further material available with which to test for the common predisposing inversion polymorphism of $8 \mathrm{p} 23.1^{1}$ The karyotype of the mother was: 46,XX, $\operatorname{dup}(8)(\mathrm{p} 22 \mathrm{p} 23.1) \mathrm{dn} . \mathrm{ish} \operatorname{dup}(8)(\mathrm{p} 22 \mathrm{p} 23.1) \quad(2629 \mathrm{I} 16+$, 122N11enh,211C9++,112G9++, 589N15,$++ 24 \mathrm{D} 9+++$, $92 \mathrm{C} 1++, 433 \mathrm{~L} 7+)$.arr $\quad \mathrm{cgh}(\mathrm{B} 35: \mathrm{CHR} 8: 7$ 256 229-12 285 $464++, 12626674-13127400+++$ ).

Family 2 G-banded analysis of peripheral blood cultures from the proband and mother showed a duplication of the 

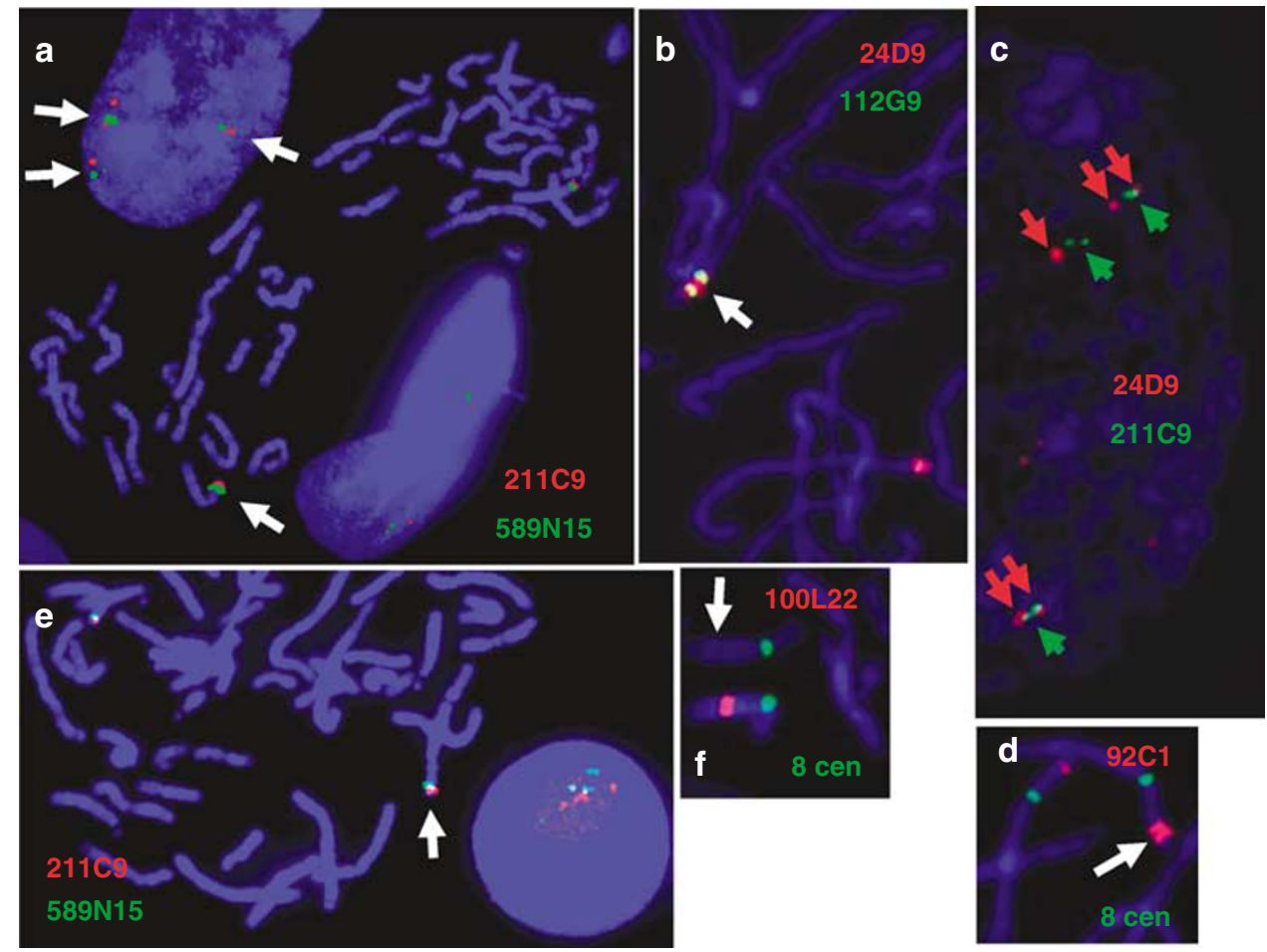

Figure 3 (a-f) Representative dual-colour FISH in Case 1, Family 1 and Family 2: (a) Case 1 - duplication of BACs which map to each end of the interval between the ORDRs in 8p23.1. Note the enlarged signals on the duplicated metaphase chromosome (white arrow) and the clear pair of extra signals in the interphase nucleus (white arrows); (b, $\mathbf{c}$ and d) Family 1 - duplication of BACs which map within the interval between ORDRs, triplication of REPD BAC 24D9 and additional duplication of region proximal to REPP: (b) note the green duplicated BAC 112G9 signals at metaphase on an extended background of red REPD BAC 24D9 signal (white arrow) and (c) the 24D9 signals (red arrows) alternating with the duplicated 211C9 signals (green arrows) at interphase; (d) confirmation of the additional duplication of proximal 8p23.1 (white arrow) with BAC 92C1 (red). (e and f) Family 2 duplication of BACs which map within the interval between ORDRs and confirmation of the additional duplication of the region proximal to REPP: (e) note the larger signals from both $211 \mathrm{C} 9$ (red) and 589N15 (green) on the duplicated metaphase chromosome (white arrow) and the three signals of each type at interphase in the proband from Family 2; (f) confirmation of the 8q22.1 deletion (white arrow) using BAC $100 \mathrm{~L} 22$ (red) in the mother from Family 2.

distal short arm of chromosome 8 (Figure 2c). Dual-colour FISH showed that BACs between REPP and REPD were duplicated (Table 1; Figure 3e). All other probes gave normal results. OaCGH in the proband and mother not only confirmed duplication of the interval between REPP and REPD but also showed that the flanking ORDRs were reduced in copy number compared to the control DNA (Figure $4 \mathrm{a}$; Table 1). A further unexpected deletion of $3.14 \mathrm{Mb}$ from $8 \mathrm{q} 22.1$ was found in both mother and proband (Figure 4b; Table 1) and confirmed using FISH with BACs RP11-100L22 and RP11-10N23 (Figure 3f; Table 1). The same polymorphism within introns $4-5$ of the GATA4 gene, as seen in Family 1, was identified with the oligonucleotide mapping to base pair 11604907 in both mother and son. No other significant gains or losses were found. The duplicated chromosome was also present in the proband's brother who died suddenly at home at the age of 7 weeks, but no DNA was available with which to test for the additional deletion of 8q22.1. The karyotype of the proband was:
46,XY,dup(8)(p23.1p23.1)del(8)(q22.1q22.1)mat.ish dup (8)del(8) $\quad(991 \mathrm{O} 23+, 2629 \mathrm{I} 16+, 122 \mathrm{~N} 11+, 211 \mathrm{C} 9++$, $112 \mathrm{G} 9++, 589 \mathrm{~N} 15++, 433 \mathrm{~L} 7+, 100 \mathrm{~L} 22-, 10 \mathrm{~N} 23-)$. arr cgh dup(8)del(8)(B35:CHR8:8 168040-11 $897580++$, 93 458057 $-96599593-)$.

\section{Molecular genetics}

Multiplex ligation-dependent probe amplification analysis with both the PO36 + (see Methods) and P139 probe kits confirmed a duplication between REPP and REPD in the proband and mother from both Families 1 and 2 (Table 1). Altered copy number of the flanking ORDRs coincided in both Families 1 and 2 with 'duplication' at both REPP and REPD in Family 1 and a corresponding 'deletion' or normal copy number at REPP and REPD in Family 2 (Table 1). However, the MLPA signals for most of the chromosome 8 probes in the P139 kit are artificially reduced with a competitor system, hence an accurate total copy number cannot be guaranteed, especially as our MLPA analysis method compares the test patient to a normal individual 
a

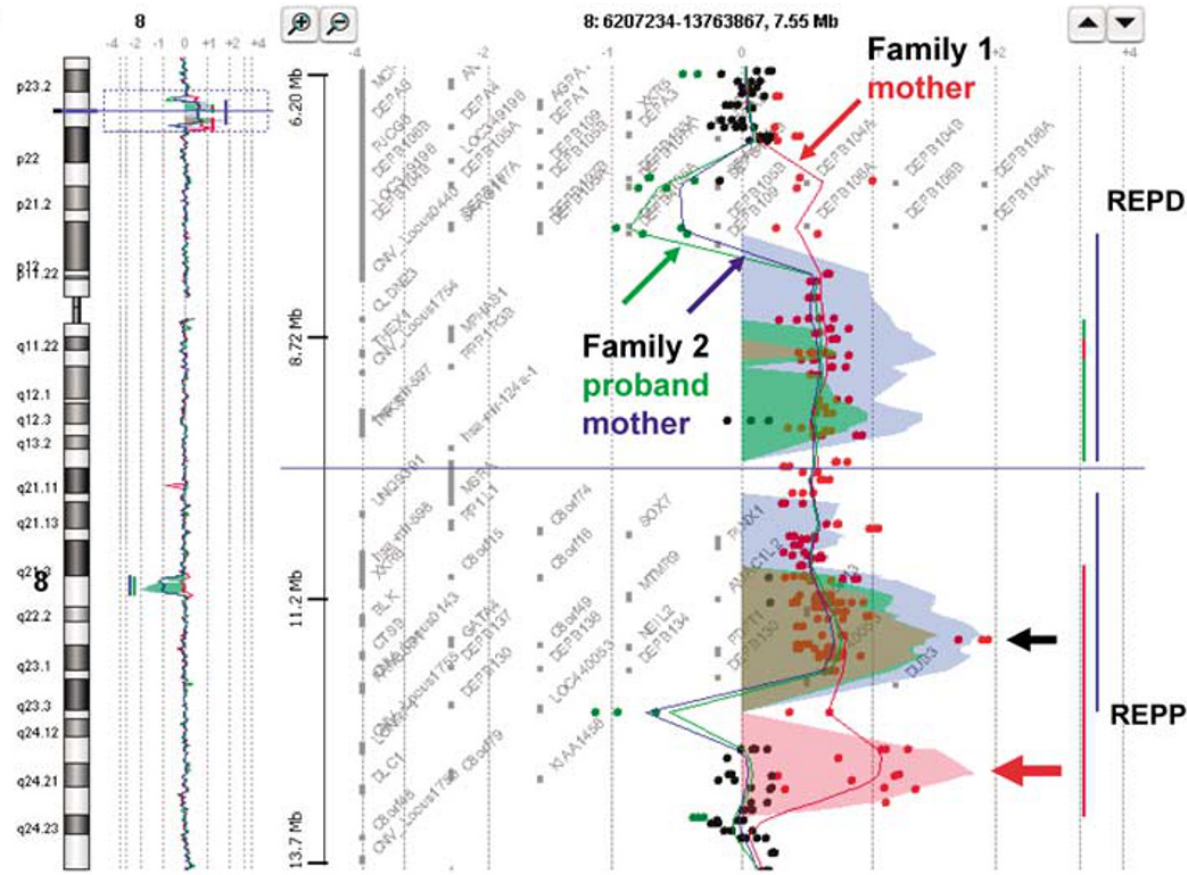

b
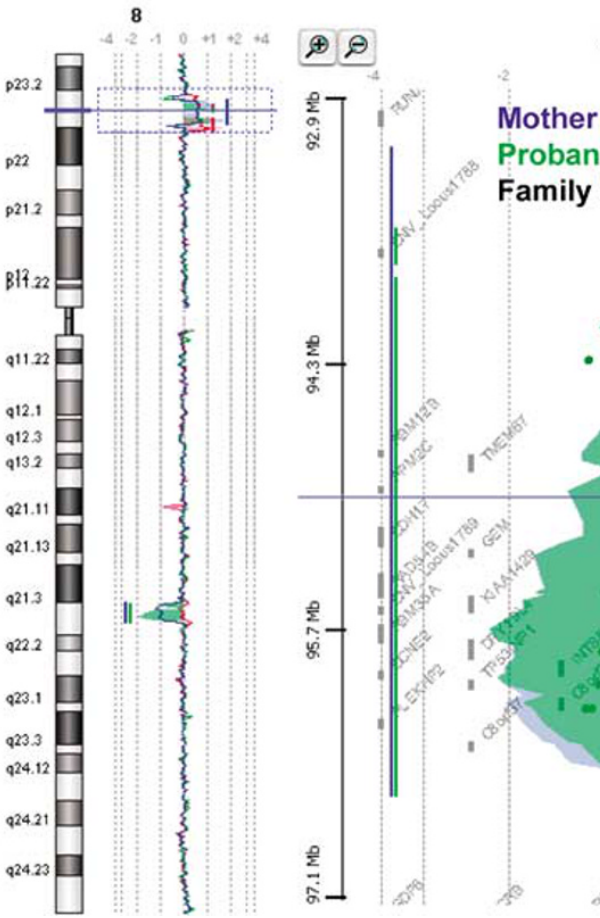

8: 92915043-97162588, 4.24 Mb

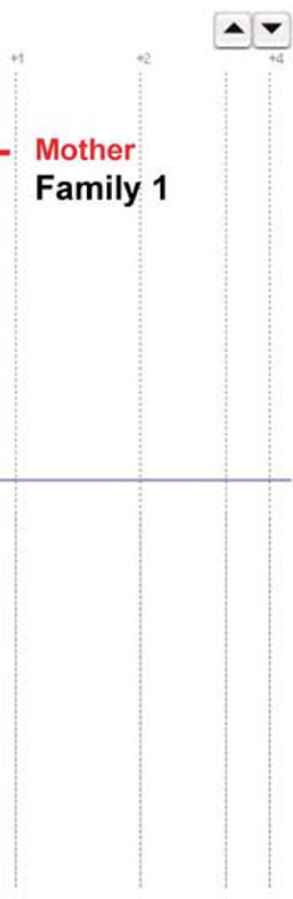

Figure 4 (a-b) Composite oligonucleotide array CGH results analysed using Agilent Analytics 3.4 software in the mother from Family 1 (red track), the proband from Family 2 (green track) and the mother from Family 2 (blue track): (a) note the consistent relative decrease in oligonucleotide copy number of the REPD variable defensin cluster in Family 2 (green and blue tracks) opposite the relative increase in Family 1 (red track), the consistent duplications extending from REPP to REPD (red, green and blue tracks), the consistent relative decrease in oligonucleotide copy number of the REPP variable defensin cluster in Family 1 (green and blue track) opposite the relative increase in Family 2 (red track), the additional triplication proximal to REPP (large red arrow) in the mother of Family 1 (red track only) and the GATA4 introns 4-5 copy number variation (slim black arrow); (b) note the consistent deletions in the proband from Family 2 (green track) and the mother from Family 2 (blue track) in contrast with the normal copy number of the mother of Family 1 (red track). Please note also that the horizontal coloured peaks are automatically generated by the software and only approximate indicators of the extent of the duplications and deletions delineated by the individual coloured spots of relative oligonucleotide signal strength. 
who may have a variable copy number of one or more of the defensin genes. No evidence of the REPP to REPD duplication was found in the maternal grandmother from Family 2. DNA was not available from the grandfather.

\section{Discussion}

We have presented five additional prenatal and transmitted familial cases which all contain a core $\sim 3.75 \mathrm{Mb}$ duplication of most of band $8 \mathrm{p} 23.1$ between the proximal (REPP) and distal (REPD) ORDRs. Excluding REPP and REPD, the duplicated interval between them contains 57 genes of which 34 are known and 23 are novel. These include the two transcription factors GATA4 and SOX7 and three micro-RNA loci. OaCGH revealed an additional duplication of at least $500 \mathrm{~kb}$ extending proximally from REPP into 8p22 in Family 1 (Figure 4a). This interval contains three further genes and interrupts the Deleted in Liver Cancer gene (DLC1) at introns 5-6 of isoform 1. OaCGH also identified an additional deletion of at least $3.1 \mathrm{Mb}$ from the Nablus Mask-Like Facial Syndrome (NMLFS) region in $8 \mathrm{q} 22.1^{18}$ in Family 2 (Figure $4 \mathrm{~b}$ ). This interval contains a further 26 genes of which six are known and 18 are novel.

These imbalances provide evidence for a new microduplication syndrome with a variable phenotype. Case 1 has only the characteristic prominent forehead and arched eyebrows. She is developmentally normal at the age of 15 months. Development appears to be delayed in some individuals from Families 1 and 2 but our adult patients live independent lives with varying degrees of support. The phenotype in the proband of Family 1 is complicated by the severe primary adrenal insufficiency with which she presented. The absence of a history of adrenal insufficiency in the mother and other patients, together with the lack of obvious candidate genes, suggests that it is the adrenal insufficiency that brought this girl to medical attention at such a young age and that this may be either a rare complication or a co-incidental finding. Her TOF is, however, consistent with the idea that GATA4 is a dosagesensitive heart disease gene with variable penetrance. ${ }^{7}$ The mother had the characteristic facial features but no known evidence of a heart defect. The additional duplication in this family includes the peptidase LONRF1, the candidate tumour suppressor gene KIAA1456 and the hypothetical protein-coding gene C8orf79. LONRF1 overlaps with Redon Copy Number Polymorphism cnp684 and cnp 685 overlaps with both KIAA1456 and C8orf79. The duplication also interrupts $D L C 1$ but neither this candidate hepatocellular tumour suppressor nor the other genes are good candidates for any of the phenotypic features described in Family 1.

The phenotype in Family 2 was regarded as more severe and included a greater degree of learning difficulties, cleft palate and partial $2 / 3$ toe syndactyly. This was also the family with the additional 8q22.1 deletion of $3.14 \mathrm{Mb}$ within the $4.2 \mathrm{Mb}$ NMLFS critical region. ${ }^{18}$ A diagnosis of NMLFS had been considered before the array CGH results but thought unlikely in the light of the 8p23.1 duplication and Family 2's less severe features. The two previously reported NMLFS cases had a similar, but more extreme, phenotype to our Family 2, with both patients having a greater degree of blepheraphimosis, distinctive dysplastic ears and one having a submucosal cleft palate. However, the NMFLS patients had a number of other features including multiple joint contractures, microcephaly and an appearance of glistening skin that were lacking in Family $2 .{ }^{18}$ Thus, we may have ascertained a more mildly affected NMLFS family because of the independent duplication of 8 p23.1 or these other additional features may be due to haploinsufficiency of genes within the larger $4.2 \mathrm{Mb}$ interval. An analogous situation has been reported in cri-duchat syndrome where array CGH consistently revealed additional imbalances in patients with a more severe phenotype. ${ }^{19}$ The novel introns 4-5 GATA4 copy number variation detected in all three arrayed individuals was also present in other unrelated patients (data not shown) but could conceivably have an effect on expression or splicing of the GATA4 gene.

The closest precedent in the literature was an 8-year-old girl with a de novo duplication of $8 \mathrm{p} 23.1$ between the ORDRs alternating with three copies of a $300-450 \mathrm{~kb}$ segment distal to REPD. ${ }^{7}$ She had mild language delay, pulmonary stenosis, height and head circumference on the 97th centile, difficulties with concentration, perception and response, and sensitivity to noise. She was not markedly dysmorphic but did have prominent brow ridges and long arched eyebrows. A duplication of the same interval with a much larger distal triplication between REPD and the MYOM2 repeats in $8 \mathrm{p} 23.3$ was recently reported. ${ }^{10}$ Case 2 of Giorda et al ${ }^{10}$ was said to resemble the de novo patient of Barber et $\mathrm{al}^{7}$ and their Case 1 to give an impression of Kabuki syndrome because of long arched eyebrows as did a further patient with a de novo triplication of $8 \mathrm{p} 22-23 .^{20}$ This suggests that the interval between REPP and REPD does contain the gene(s) for a facial phenotype with arched eyebrows reminiscent of, but not consistent with, classical Kabuki syndrome. In addition, our patients lack the long eyes and everted lower eyelids of Kabuki syndrome. Further cytogenetic duplications of 8p23.1 have been reported but not analysed using FISH or array $\mathrm{CGH}^{21,22} \mathrm{~A}$ variable phenotype including speech delay, minor facial dysmorphisms and heart defects are a recurring theme among the larger transmitted duplications extending from $8 \mathrm{p} 23.1$ distally, $^{23}$ proximally $^{24-26}$ or both. $^{27}$

Altered copy number of the flanking ORDRs coincided in both Families 1 and 2 with Family 1 having 'duplications' at REPP and REPD and Family 2 having corresponding 'deletions' at REPP and REPD using MLPA and array CGH 
(Figure $4 \mathrm{a}$ and $\mathrm{b}$ ). Total copy number of these regions is known to vary from 2 to 7 in the normal population ${ }^{12}$ and only reduction in medial copy number from 4 to 3 has been associated with predisposition to Crohn's disease. ${ }^{28}$ However, absolute ORDR copy number has not been determined in the present cases due to the 'competitor' system used in the P139 kit and the fact that copy number in both MLPA and CGH has only been determined relative to controls with unknown defensin copy numbers. Nevertheless, it is most likely that the 8 p23.1 microduplication syndrome is a further genomic disorder mediated by flanking ORDRs because (1) there is a common core duplication between REPP and REPD in all cases, (2) the 8p23.1 duplication is confined to this interval in Family 2, (3) the duplication in Family 1 alternates between three copies of REPP (Figures $3 b$ and c) and (4) the core duplication alternated between three copies of a 300$450 \mathrm{~kb}$ segment adjacent to REPD in the previously reported case. ${ }^{7}$ An analogous situation has been described in Pelizaeus-Merzbacher disease, where genomic architecture underlies a variety of duplications all of which contain the PLP1 gene. ${ }^{29}$ However, there were no evident segmental duplications or copy number variations at the additional 8 p22 or 8 q22.1 breakpoint regions according to the Structural Variation Database or the Database of Genomic Variants. Despite the flanking repeats, this 8p23.1 duplication syndrome appears to be rare as it has not yet, to our knowledge, been found using tiling path ${ }^{5}$ or elegant applications of array CGH targeted at regions flanked by homologous repeats in large numbers of patients. ${ }^{6}$ Alternatively, the mild phenotype in some patients may mean that the condition is underascertained.

In conclusion, we describe the variable features in five patients with the 8p23.1 duplication syndrome and find a characteristic facial phenotype with a prominent forehead and arched eyebrows. Early childhood development can be normal but development is delayed in some individuals. There is marked variability in the congenital anomalies associated with this new duplication syndrome, only some of which may be related to the additional imbalances found. Our results demonstrate that direct transmission from a parent does not discriminate between genuine duplications and euchromatic variants and illustrate the power of array CGH to reveal unexpected additional genomic imbalances in affected patients.

\section{Acknowledgements}

We are very grateful to the families involved for contributing additional samples, to Laura Yates, Alison Stewart and Sharon Mcdonnell for their help in gathering information about Family 1 and to $\mathrm{Dr} C$ Dorche for the sulphite oxidase testing. This work could not have been carried out without the $1 \mathrm{Mb}$ and $37 \mathrm{k}$ cloneset BACs provided kindly by the Sanger Institute of Hinxton, Cambridge, UK. VKM and SH are financially supported as part of the National Genetics Reference Laboratory (Wessex) by the UK Department of
Health. The image enhancement equipment used for parts of this work was provided by the Welcome Trust and the Trust Funds of Salisbury NHS Foundation Trust.

\section{URLs}

Ensembl tiling path (http://www.ensembl.org/homo_sapiens/ cytoview),

MartView data export tool (www.biomart.org/biomart/martview/), Database of Genomic Variants (http://projects.tcag.ca/variation/), Structural Variation Database (http://humanparalogy.gs.washington. edu/structuralvariation/).

\section{References}

1 Giglio S, Broman KW, Matsumoto $\mathrm{N}$ et al: Olfactory receptor-gene clusters, genomic-inversion polymorphisms, and common chromosome rearrangements. Am J Hum Genet 2001; 68: 874-883.

2 Devriendt K, Matthijs G, Van Dael R et al: Delineation of the critical deletion region for congenital heart defects on chromosome 8p23.1. Am J Hum Genet 1999; 64: 1119-1126.

3 Shimokawa O, Miyake N, Yoshimura T et al: Molecular characterization of $\operatorname{del}(8)(\mathrm{p} 23.1 \mathrm{p} 23.1)$ in a case of congenital diaphragmatic hernia. Am J Med Genet A 2005; 136: 49-51.

4 Slavotinek A, Lee SS, Davis R et al: Fryns syndrome phenotype caused by chromosome microdeletions at 15q26.2 and 8p23.1. J Med Genet 2005; 42: 730-736.

5 de Vries BB, Pfundt R, Leisink M et al: Diagnostic genome profiling in mental retardation. Am J Hum Genet 2005; 77: 606-616.

6 Sharp AJ, Hansen S, Selzer RR et al: Discovery of previously unidentified genomic disorders from the duplication architecture of the human genome. Nat Genet 2006; 38: 1038-1042.

7 Barber JCK, Maloney V, Hollox EJ et al: Duplications and copy number variants of 8 p23.1 are cytogenetically indistinguishable but distinct at the molecular level. Eur J Hum Genet 2005; 13: $1131-1136$.

8 Giglio S, Calvari V, Gregato G et al: Heterozygous submicroscopic inversions involving olfactory receptor-gene clusters mediate the recurrent t $(4 ; 8)(\mathrm{p} 16 ; \mathrm{p} 23)$ translocation. Am J Hum Genet 2002; 71: 276-285.

9 Sugawara H, Harada N, Ida T et al: Complex low-copy repeats associated with a common polymorphic inversion at human chromosome 8p23. Genomics 2003; 82: 238-244.

10 Giorda R, Ciccone R, Gimelli G et al: Two classes of low-copy repeats comediate a new recurrent rearrangement consisting of duplication at 8p23.1 and triplication at 8p23.2. Hum Mutat 2007; 28: $459-468$

11 Barber JC: Directly transmitted unbalanced chromosome abnormalities and euchromatic variants. J Med Genet 2005; 42: $609-629$.

12 Hollox EJ, Armour JAL, Barber JCK: Extensive normal copy number variation of a $\beta$-defensin antimicrobial gene cluster. Am J Hum Genet 2003; 73: 591-600.

13 Taudien S, Galgoczy P, Huse K et al: Polymorphic segmental duplications at 8 p23.1 challenge the determination of individual defensin gene repertoires and the assembly of a contiguous human reference sequence. BMC Genomics 2004; 5: 92.

14 Aldred PM, Hollox EJ, Armour JA: Copy number polymorphism and expression level variation of the human alpha-defensin genes DEFA1 and DEFA3. Hum Mol Genet 2005; 14: 2045-2052.

15 Seebah S, Suresh A, Zhuo S et al: Defensins knowledgebase: a manually curated database and information source focused on the defensins family of antimicrobial peptides. Nucleic Acids Res 2007; 35: D265-D268.

16 Armour JA, Palla R, Zeeuwen PL, den Heijer M, Schalkwijk J, Hollox EJ: Accurate, high-throughput typing of copy number 
variation using paralogue ratios from dispersed repeats. Nucleic Acids Res 2007; 35: e19.

17 Schouten JP, McElgunn CJ, Waaijer R, Zwijnenburg D, Diepvens F, Pals G: Relative quantification of 40 nucleic acid sequences by multiplex ligation-dependent probe amplification. Nucleic Acids Res 2002; 30: e57.

18 Zhang X, Snijders A, Segraves R et al: High-resolution mapping of genotype-phenotype relationships in cri du chat syndrome using array comparative genomic hybridization. Am J Hum Genet 2005; 76: 312-326.

19 Shieh JT, Aradhya S, Novelli A et al: Nablus mask-like facial syndrome is caused by a microdeletion of $8 \mathrm{q}$ detected by arraybased comparative genomic hybridization. Am J Med Genet A 2006; 140A: 1267-1273.

20 Shieh JT, Hudgins L, Cherry AM, Shen Z, Hoyme HE: Triplication of $8 \mathrm{p} 22-8 \mathrm{p} 23$ in a patient with features similar to Kabuki syndrome. Am J Med Genet A 2006; 140A: 170-173.

21 Kennedy SJ, Teebi AS, Adatia I, Teshima I: Inherited duplication, $\operatorname{dup}(8)(\mathrm{p} 23.1 \mathrm{p} 23.1) \mathrm{pat}$, in a father and daughter with congenital heart defects. Am J Med Genet 2001; 104: 79-80.

22 Tsai C-H, Graw SL, McGavran L: 8p23 Duplication reconsidered: is it a true euchromatic variant with no clinical manifestation? J Med Genet 2002; 39: 769-774.
23 Engelen JJM, Moog U, Evers JLH, Dassen H, Albrechts JCM, Hamers AJH: Duplication of chromosome region 8 p23.1 $\rightarrow$ p23.3: a benign variant? Am J Med Genet 2000; 91: 18-21.

24 Dhooge C, Vanroy N, Craen M, Speleman F: Direct transmission of a tandem duplication in the short arm of chromosome 8 . Clin Genet 1994; 5: 36-39.

25 Engelen JJM, de Die-Smulders CEM, Sijstermans JMJ, Meers LEC, Albrechts JCM, Hamers AJH: Familial partial trisomy 8p without dysmorphic features and only mild mental retardation. $J$ Med Genet 1995; 32: 792-795.

26 Fan Y-S, Siu VM, Jung JH, Farrell SA, Cote GB: Direct duplication of $8 \mathrm{p} 21.3-\mathrm{p} 23.1$ : a cytogenetic anomaly associated with developmental delay without consistent clinical features. Am J Med Genet 2001; 103: 231-234.

27 Brooks SS, Genovese M, Gu H, Duncan CJ, Shanske A, Jenkins EC: Normal adaptive function with learning disability in duplication 8p including p22. Am J Med Genet 1998; 78: 114-117.

28 Fellermann K, Stange DE, Schaeffeler E et al: A chromosome 8 gene-cluster polymorphism with low human beta-defensin 2 gene copy number predisposes to Crohn disease of the colon. Am J Hum Genet 2006; 79: 439-448.

29 Lee JA, Inoue K, Cheung SW, Shaw CA, Stankiewicz P, Lupski JR: Role of genomic architecture in PLP1 duplication causing PelizaeusMerzbacher disease. Hum Mol Genet 2006; 15: 2250-2265. 